\title{
Phase-slip memory effects in dissipation-free superflow
}

\author{
K. Schwab, J. Steinhauer, and Richard Packard \\ Physics Department, University of California, Berkeley, California 94720
}

(Received 3 December 1996)

\begin{abstract}
Critical superflow, preceding a free ringing superfluid oscillation, induces a remnant stochastic uncertainty in the oscillation amplitude. We demonstrate that the distribution function for the oscillation's velocity amplitude reflects both the quantized size of the underlying phase-slip dissipation events, as well as the stochastic nature of the processes which nucleate the slips [S0163-1829(97)04014-9]
\end{abstract}

Flow in superfluid ${ }^{4} \mathrm{He}$ is often without dissipation below some critical velocity. Anderson ${ }^{1}$ suggested that above this critical velocity, the dissipative process involves the creation and subsequent motion of quantized vortex filaments. He showed that if such a filament crosses the streamlines passing through a restricted passage, the energy in the flow will decrease by a fixed amount, corresponding to a $2 \pi$ decrease in the quantum phase difference across the passage. Recently it has been possible to detect these individual $2 \pi$ phase-slip dissipation events in superfluid Helmholtz-type oscillators. ${ }^{2-4}$ It is the purpose of this paper to show that the discrete nature of the phase slips can also be detected in dissipation-free flow that follows dissipative flow. The dissipation-free flow contains a memory of the prior quantized dissipative flow.

A commonly used technique to study superflow involves driving fluid through a constricting path (e.g., a thin film or a small aperture) under the influence of a relaxing pressure head. ${ }^{5,6}$ A flow transient is typified by an (almost pressurehead-independent) constant critical-velocity relaxation. ${ }^{7}$ This dissipative part of the transient, is often followed by a lightly damped, oscillatory, free ringing response, which is due to the exchange between the kinetic energy in the flow and the potential energy in the driving mechanism (e.g., gravity or a stretched membrane). The initial velocity amplitude of the resulting oscillation corresponds to the critical velocity, which is the slope of the transient immediately before the oscillation begins.

The experiment described here concerns superfluid ${ }^{4} \mathrm{He}$ flowing through a submicron aperture. ${ }^{8}$ Figure 1 is a schematic diagram of the basic apparatus which is the same as that used to study the stochastic nucleation of phase slips. ${ }^{9}$ The fabrication details are described in Ref. 10. The aperture ${ }^{11}$ couples two superfluid reservoirs: inside to outside. A flexible, metalized, Kapton diaphragm, in a partition between the reservoirs, is manipulated by the application of electrostatic forces by a nearby electrode. The diaphragm's position is monitored by detecting changes in the inductance of an adjacent superconducting coil. For small fluid velocities in the aperture, the device behaves as a simple harmonic oscillator of the Helmholtz variety, with a resonant frequency of $40.7 \mathrm{~Hz}$. The restoring force is determined by the diaphragm's tension and the inertia is dominated by the fluid moving in the aperture. The experiment is cooled by an adsorption-pumped ${ }^{3} \mathrm{He}$ refrigerator.
Figure 2 shows a typical measured transient. At the point marked A, the flexible Kapton diaphragm is pulled toward the fixed actuator electrode by the application of a voltage step. This action causes a hydrostatic pressure head, $\Delta P$, to appear across the aperture. The resultant superflow is then recorded by monitoring the diaphragm's motion with the sensitive superconducting quantum interference devicebased displacement transducer. The superflow shows a nearly constant velocity relaxation (B), and a free ringing oscillation (C). We find that as a result of the prior dissipative flow, this free ringing amplitude will be uncertain by an amount that reflects both the quantized nature of the phase slippage and the stochastic nature of the vortex nucleation process.

Discrete phase slips are nucleated stochastically when the superfluid velocity approaches a critical velocity. ${ }^{12}$ The flow through the aperture is expected to evolve in a manner similar to that shown in Fig. 3. Figure 3(a) shows the velocity versus time of a calculated transient, ${ }^{13}$ where the diaphragm position versus time is essentially identical to that of Fig. 2 . At the point marked $\mathrm{A}$, the driving pressure is applied and the superfluid quickly accelerates. At point $\mathrm{B}$, the velocity has reached the critical velocity. The "hair" in the subsequent period of time comes from the quantized dissipation. The fluid is alternately accelerated due to the (diminishing) pressure, head and then abruptly decelerated as a quantized vortex is nucleated and then passes across the aperture. Finally, at point $\mathrm{C}$, (almost) all of the initial potential energy

A

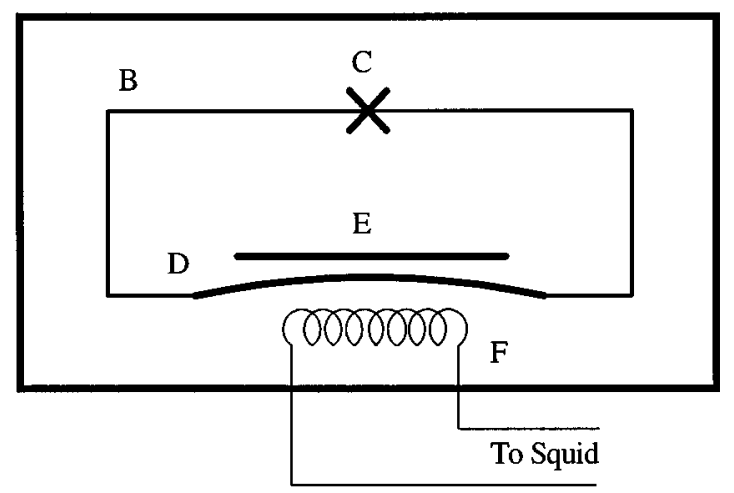

FIG. 1. Cell schematic: A is the outer brass box filled with superfluid ${ }^{4} \mathrm{He}, \mathrm{B}$ is the inner silicon box, $\mathrm{C}$ is the aperture, $1.25 \mu$ $\times 0.38 \mu \mathrm{m}, \mathrm{D}$ is the flexible, $8 \mu \mathrm{m}$ thick, Kapton diaphragm, $\mathrm{E}$ is the electrode, $\mathrm{F}$ is the superconducting coil displacement sensor. 


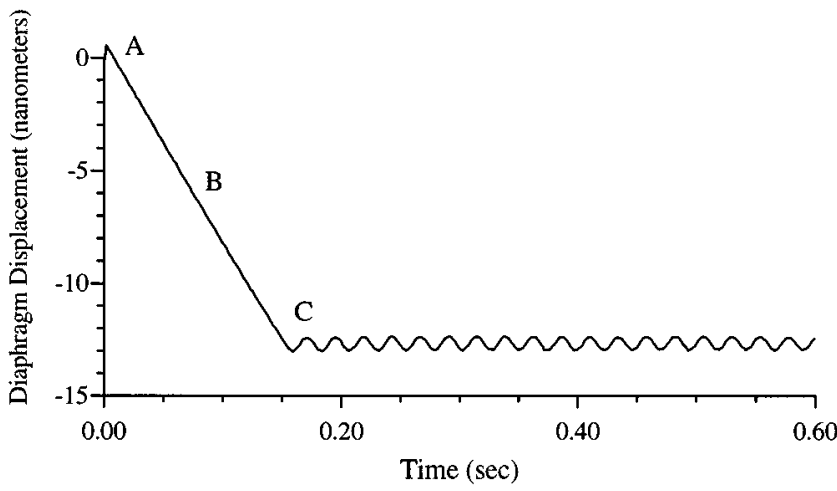

FIG. 2. Typical recorded transient at a temperature of $0.61 \mathrm{~K}$ : A indicates the sudden application of driving pressure, B is nearconstant velocity superflow, $\mathrm{C}$ indicates free ringing.

has been dissipated, and the free ringing begins. Figure 3(b) shows an expanded picture near the onset of the oscillation.

The discrete $2 \pi$ dissipation events are triggered by stochastic processes (thermal ${ }^{14}$ or quantum ${ }^{15,16}$ ) each time the superfluid velocity in the aperture approaches the critical value $v_{c}$. Each phase slip results in a decrease in the flow energy,

$$
\delta E=\kappa_{o} \rho_{s} v_{c} a,
$$

where $\kappa_{o}=h / m_{4}$ is the circulation quantum, $\rho_{s}$ is the superfluid density, and $a$ is the area of the aperture. The average rate of these phase slips is given by the Josephson-Anderson frequency, $f_{\mathrm{JA}}=\Delta / P \rho \kappa_{o}$, where $\Delta P$ is the instantaneous pressure difference across the aperture.

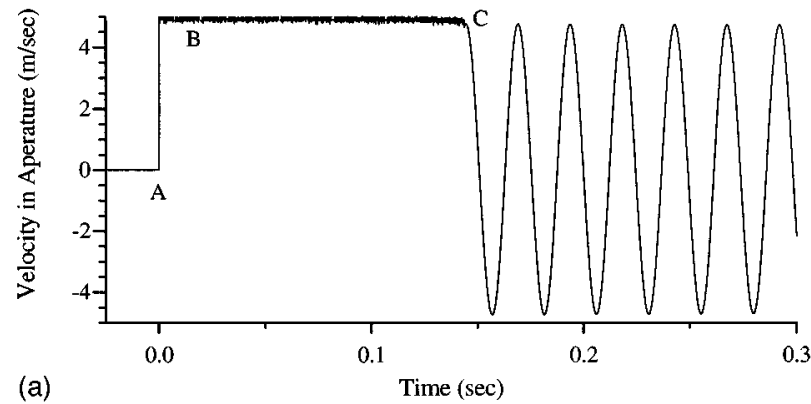

(a) Time (sec)

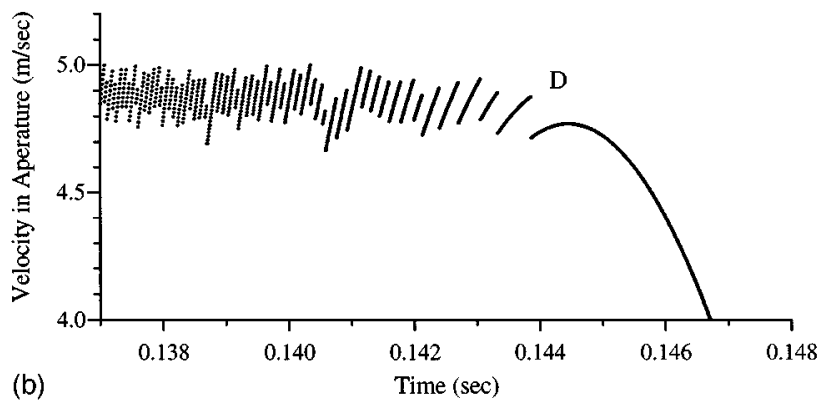

FIG. 3. Simulated time evolution of the superfluid velocity in the aperture. (a) shows the entire transient: A is the application of driving force, $\mathrm{B}$ is critical velocity reached and phase-slip dissipation begins, $\mathrm{C}$ indicates driving pressure exhausted and free ringing Helmholtz oscillation begins. Details of phase slippage and transition is shown in (b): D marks transition to free ringing oscillation.

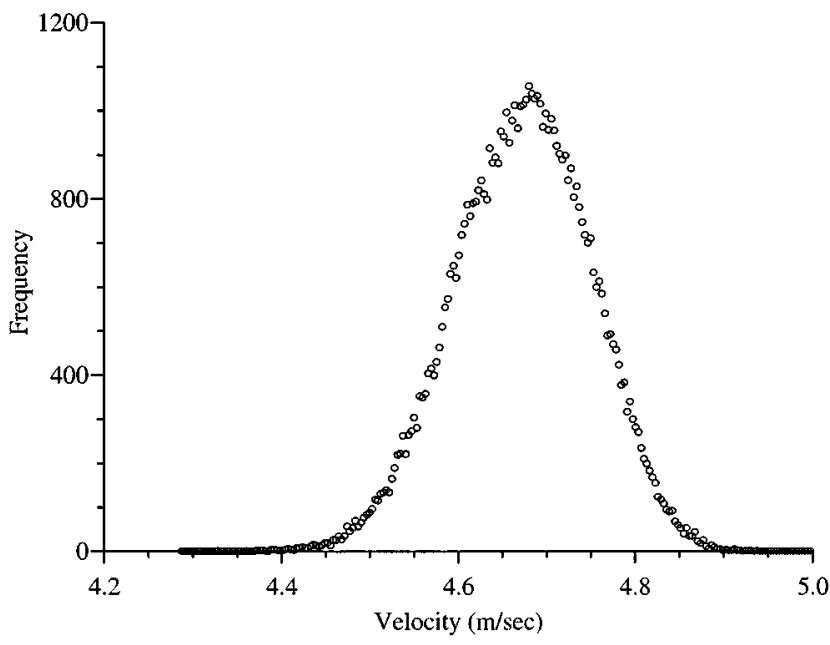

FIG. 4. Distribution function of phase-slip critical velocity measured by observing individual dissipation events.

As the diaphragm approaches its final equilibrium position, the pressure head steadily diminishes with a corresponding decrease in phase-slip frequency. Finally, there is one last phase slip occurring at some instant, marked D in Fig. 3(b). This is followed by the damped oscillatory response of the, now linear, Helmholtz oscillator. The subsequent damping of this oscillation is due primarily to linear, thermal-mechanical dissipation. ${ }^{17}$

The phase slips induce an uncertainty in the oscillation amplitude in two ways. The dissipation is quantized, but the total initial potential energy is a continuous function of the initial applied voltage step. After the last slip, the system is left with some small residual potential energy. This energy is not sufficient to accelerate the superfluid to the critical value. However, there is an uncertainty in the energy dissipated in each slip. This is a result of the distribution of the phase-slip critical velocity (typically $\sigma / v_{c}$ is about $2 \%$ ) arising from the stochastic nature of the vortex nucleation process [see Eq. (1)]. Consequently the residual energy, after many phase slips, ${ }^{18}$ will have a rectangular-shaped distribution function which is is bounded by the size of one phase slip.

In addition to the distribution in oscillation amplitudes caused by the quantized nature of the dissipation, there is also an effect from the stochastic nucleation of the individual phase slips. Because of the stochastic nucleation mechanisms, both thermal and quantum, the individual slips occur with a distribution of critical velocities. The uncertainty in the velocity which nucleates the final slip will have the effect of spreading the rectangular distribution caused by the quantization of the dissipation. Thus, if many oscillation transients are recorded, a histogram of the initial oscillation amplitudes should be determined by a convolution of the finite quantization and the stochastic nucleation.

In our experiment we can directly measure both the stochastic distribution function for phase-slip critical velocities and the phase-slip size. We use these numbers as input parameters for the numerical simulation of a flow transient such as that shown in Fig. 2. By repeating the simulation many times, we calculate the expected distribution in Helmholtz oscillation amplitudes. We compare this prediction to the measured distribution of oscillation amplitudes of 350 actual recorded flow transients.

The distribution function of the individual phase slips is measured by driving the Helmholtz oscillation on resonance 
with a fixed amplitude driving force, applied through the actuator electrode. For temperatures below $1 \mathrm{~K}$, the $Q$ of the oscillator is quite high, often greater than $10^{4}$. As the amplitude grows in time, one can plot the amplitude of alternate half cycles. When a single phase slip occurs the oscillation amplitude is seen to abruptly drop. ${ }^{2,3}$ We can thus record the critical oscillation amplitude, $X_{c}$, at which a single phase slip occurs. This corresponds to a critical velocity $v_{c}=\omega X_{c}$. By observing thousands of slips one can make a histogram of the critical velocity values. A typical phase-slip distribution function, measured at $0.57 \mathrm{~K}$, is shown in Fig. 4 . At this temperature we find the parameters $v_{x}=4.67 \mathrm{~m} / \mathrm{s}$ and $\sigma=0.079 \mathrm{~m} / \mathrm{s}$, with a slip size of $0.17 \mathrm{~m} / \mathrm{s}$. The displacement transducer noise in these measurements produces an uncertainty in each measurement of $v_{c}$ of $0.004 \mathrm{~m} / \mathrm{s}$. This uncertainty is negligible compared to the intrinsic stochastic uncertainty.

Using these measured phase-slip distribution parameters, we predict the distribution function for free ringing Helmholtz oscillation amplitudes. We do this by inserting these parameters into the numerical simulation in which we integrate the equation of motion of the diaphragm with the addition of quantized, phase-slip dissipation. This dissipation is stochastically distributed with the above parameters, $v_{c}, \sigma$, and phase-slip size. The predicted distribution of 20000 iterations of this simulation is shown as the solid line in Fig. 5.

The Helmholtz oscillation distribution function is measured as follows. The free ringing oscillation is excited $(T=0.61 \mathrm{~K})$ by applying a large fixed step potential to the actuator electrode ( $V=19.1$ volts, corresponding to an initial driving pressure $\Delta P=4.5 \mathrm{mPa}$, and a final diaphragm displacement of $12 \mathrm{~nm}$ ). A transient like that shown in Fig. 2 results. Since the $Q$ of the oscillator is large, $\sim 2000$, one can determine the initial amplitude of the free ringing oscillations with high precision. We do this by digitally recording an oscillation for $1.24 \mathrm{~s}$, sampling every $1.46 \mathrm{~ms}$. Then we calculate the Fourier transform of the free ringing oscillation and integrate the power around the Helmholtz frequency to calculate the amplitude of the oscillation. ${ }^{19}$ With a free ringing amplitude of $2.4 \times 10^{-10} \mathrm{~m}$, we have a signal to noise of $\sim 500$. This is quite adequate to resolve the underlying statistical fluctuations in the amplitude.

By analyzing many transients we make the histogram of the initial oscillation amplitude. By multiplying the initial amplitudes by the angular frequency we obtain the histogram

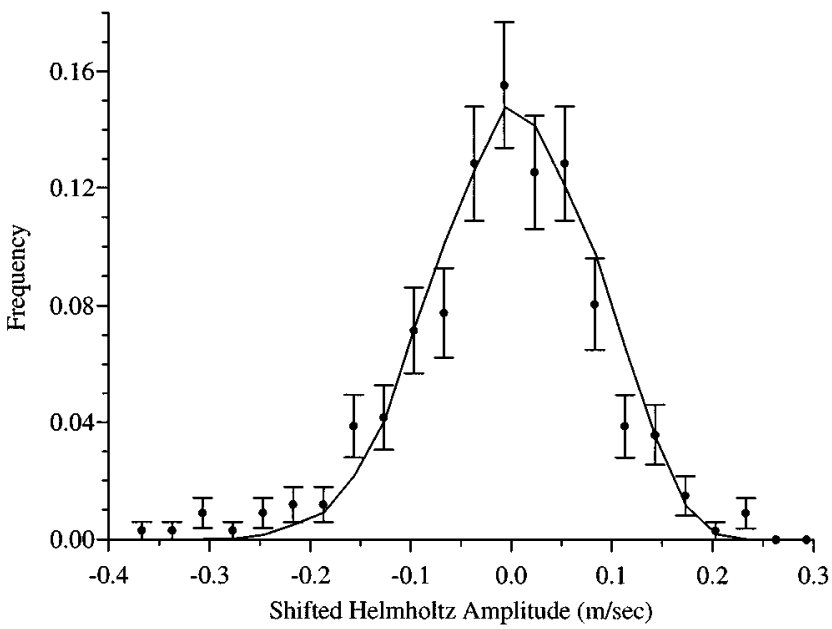

FIG. 5. Distribution of initial oscillation amplitudes. The solid line is predicted from the simulation using the measured phase-slip parameters. The circles are direct measurements from free ringing transients. Both curves have been shifted to allow comparison of the distribution shape.

of the initial velocity amplitude. To compare this measurement to the simulation prediction we slightly shift the mean of the measured distribution to accommodate the temperature difference ${ }^{20}$ between that of the discrete phase-slip data $(0.57$ $\mathrm{K})$ and that of the flow transients $(0.61 \mathrm{~K})$. The final measured distribution is shown in Fig. 5 plotted on top of the predicted distribution function.

It is apparent that the predicted and measured distribution functions are the same. This demonstrates that, as explained above, the amplitude of the free ringing oscillations reflects both the quantized nature and stochastic distribution of the underlying phase slippage in the dissipative regime. This agreement provides further support for the model that relies on discrete phase slippage events as the dissipative mechanism for the seemingly constant-velocity part of the transient response. Although the rapidly occurring phase-slip events are undetected in the dissipative precursor flow, the memory of the underlying fundamental dissipation process remains in the oscillator amplitude.

We are grateful for the helpful comments and discussions with S. Davis, S. Vitale, and S. Backhaus. This work was partially supported by the National Science Foundation and the Office of Naval Research.
${ }^{1}$ P. W. Anderson, Rev. Mod. Phys. 38, 298 (1966).

${ }^{2}$ O. Avenel and E. Varoquaux, Phys. Rev. Lett. 55, 2704 (1985).

${ }^{3}$ A. Amar, Y. Sasaki, R. Lozes, J. C. Davis, and R. E. Packard, Phys. Rev. Lett. 68, 2624 (1992).

${ }^{4}$ J. A. Flaten, C. A. Lindensmith, and W. Zimmermann, Jr., J. Low. Temp. Phys. 101, 743 (1995).

${ }^{5}$ George B. Hess, Phys. Rev. Lett. 27, 977 (1971).

${ }^{6}$ J. Steinhauer, K. Schwab, Yury Mukharsky, J. C. Davis, and Richard E. Packard, Phys. Rev. Lett. 74, 5056 (1995).

${ }^{7}$ There is a weak logarithmic dependence of the critical velocity on driving pressure head; see R. E. Packard and S. Vitale, Phys. Rev. B 45, 2512 (1992).
${ }^{8} \mathrm{~A}$ recent review of aperture experiments is given, in W. Zimmermann, Jr., Contemp. Phys. 37, 219 (1996).

${ }^{9}$ J. Steinhauer, K. Schwab, Yury Mikharsky, J. C. Davis, and Richard Packard, J. Low. Temp. Phys. 100, 281 (1995).

${ }^{10}$ The cell is constructed from silicon using microfabrication and micromachining techniques. See K. Schwab, J. Steinhauer, J. C. Davis, and R. E. Packard, IEEE Microelectromech. Syst. 5, 180 (1996).

${ }^{11}$ The dimensions of the aperture used in this experiment is 1.25 $\mu \mathrm{m} \times 0.28 \mu \mathrm{m}$ and is created by $e$-beam lithography on a 100 $\mathrm{nm}$ thick silicon nitride film.

${ }^{12}$ The stochastic nature of phase slippage is described, in E. Varo- 
quaux, W. Zimmermann, Jr., and O. Avenel, in Excitations in Two and Three Dimensional Quantum Fluids, edited by A. F. G. Wyatt and H. J. Lauter (Plenum, New York, 1992).

${ }^{13}$ This transient is produced by numerically integrating the equation of motion of the diaphragm and introducing stochastic phaseslip dissipation with a critical velocity of $5.0 \mathrm{~m} / \mathrm{s}$ and a standard deviation of $0.035 \mathrm{~m} / \mathrm{s}$. For more details of simulations of this type, see K. Schwab, Ph.D. thesis, University of California, Berkeley, 1996.

${ }^{14}$ E. Varoquaux, M. W. Meisel, and O. Avenel, Phys. Rev. Lett. 57, 2291 (1986).

${ }^{15}$ J. C. Davis, J. Steinhauer, K. Schwab, Yu. M. Mukharsky, A.
Amar, Y. Sasaki, and Richard E. Packard, Phys. Rev. Lett. 69, 323 (1992).

${ }^{16}$ G. G. Ihas, O. Avenel, R. Aarts, and E. Varoquaux, Phys. Rev. Lett. 69, 327 (1992).

${ }^{17} \mathrm{~S}$. Backhaus (unpublished).

${ }^{18} \mathrm{We}$ estimate that for each transient (of some 25000 individual phase slips) there will be an uncertainty of approximately three slips.

${ }^{19}$ W. Press, S. Teukolsky, W. Vetterling, and B. Flannery, Numerical Recipes in Fortran: The Art of Scientific Computing (Cambridge University Press, Cambridge, 1992).

${ }^{20}$ This shift is consistent with the measured temperature dependence of the phase-slip critical velocity. 